\title{
MITIGACIÓN DEL RIESGO DE LAVADO DE ACTIVOS Y FINANCIACIÓN DEL TERRORISMO
}

Tatiana Dulima Zabala Leal

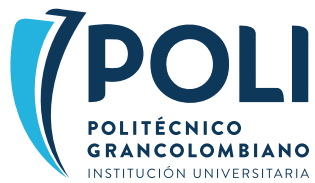


(7po

POLI

POLITÉCNICO

GRANCOLOMBIANO

INSTITUCIÓN UNIVERSITARIA

(C) Institución Universitaria Politécnico

Grancolombiano

\section{MITIGACIÓN DEL RIESGO DE LAVADO \\ DE ACTIVOS Y FINANCIACIÓN DEL TERRORISMO}

E-ISBN: 978-958-5544-37-6

\section{Editorial Politécnico Grancolombiano}

Calle 61 No. 7 - 66

Tel: 7455555, Ext. 1516

Bogotá, Colombia

Noviembre de 2019

\section{Autores}

Tatiana Dulima Zabala Leal

\section{Lider de publicaciones}

Eduardo Norman Acevedo

\section{Analista de Producción Editorial}

Carlos Eduardo Daza Orozco

\section{Corrección de Estilo \\ Hernán Dario Cadena}

Creado en Colombia
Todos los derechos reservados

No se permite la reproducción total o parcial de esta obra, ni su incorporación a un sistema informático, ni su tratamiento en cualquier forma o medio existentes o por existir, sin el permiso previo y por escrito de la Editorial de la Institución Universitaria Politécnico Grancolombiano

Para usos académicos y científicos, la Institución Universitaria Politécnico Grancolombiano accede al licenciamiento Creative Commons del contenido de la obra con: Atribución - No comercial - Sin derivar - Compartir igual

El contenido de esta publicación se puede citar o reproducir con propósitos académicos siempre y cuando se dé la fuente o procedencia

Las opiniones expresadas son responsabilidad exclusiva del autor(es) y no constituye una postura institucional al respecto.

La Editorial del Politécnico Grancolombiano pertenece a la Asociación de Editoriales Universitarias de Colombia (ASEUC) 


\section{CONTENIDO}

UNIDAD I ASPECTOS GENERALES Y DEFINICIONES

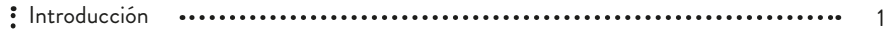

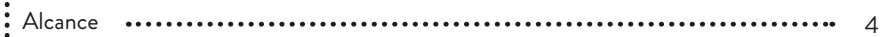

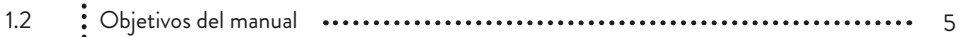

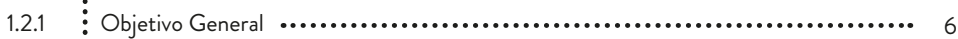

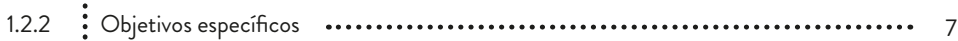

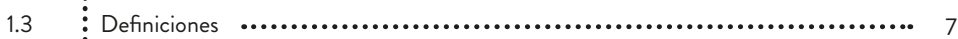

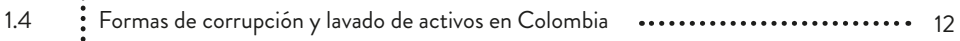

$1.5 \vdots$ Corrupción, lavado de activos y financiación del terrorismo $\ldots . . . \ldots \ldots \ldots . . . \ldots \ldots \ldots . .15$

$1.6 \quad$ Marco normativo anticorrupción en Colombia (Línea de tiempo) ................. 16

UNIDAD II ETAPAS DEL SARLAFT Pág.

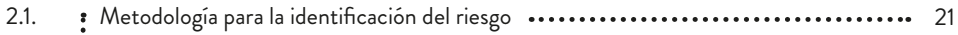

2.1.1. Juicios basados en la experiencia y los registros $\quad \ldots \ldots \ldots \ldots \ldots \ldots \ldots \ldots \ldots \ldots \ldots \ldots . . . \ldots 21$

2.1.2. $\quad$ Seguimiento de operaciones para el análisis de operaciones sospechosas.......... .22

2.1.3. $\quad$ Los procedimientos estadísticos más empleados $\quad$............................ 24

2.1.4. $\quad$ Las entradas que necesitan para realizar la segmentación $\quad$....................... 24

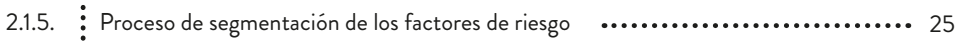

2.1.5.1. Segmentación de clientes o personal interno y/o externo $\ldots \ldots \ldots \ldots \ldots \ldots \ldots \ldots \ldots \ldots . . \ldots 25$

2.1.6. $\quad$ Medición o Evaluación $\quad$............................................... 32

2.1.6.1. Encuesta a expertos $\quad$.

2.1.6.2. Procedimiento para medir el riesgo de lavado de activos y financiación del terrorismo.. 33

2.1.7. $\quad$ Control de riesgo de lavado de activos y financiación del terrorismo $\ldots . . . \ldots \ldots \ldots \ldots . . .33$

2.1.7.1. Procedimiento para definir las medidas de control $\quad$............................ 34

2.1.8. Monitoreo del riesgo de lavado de activos y financiación del terrorismo $\quad$............. 34

2.1.8.1. Actividades para adelantar en la construcción de indicadores ..................... 35

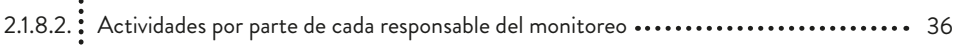

2.1.8.3. Evaluación de controles $\quad$............................................ 37 


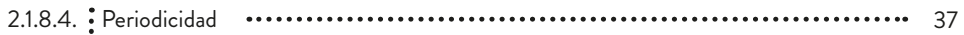

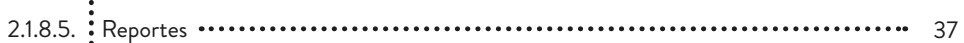

2.1.9. $\quad$ Identificación de operaciones sospechosas $\ldots \ldots \ldots \ldots \ldots \ldots \ldots \ldots \ldots \ldots \ldots \ldots \ldots \ldots \ldots . \ldots \ldots$

2.1.10. Identificación y análisis de operaciones inusuales $\quad$.

2.1.10.1. Propuesta de metodología para la detección de operaciones inusuales $\ldots \ldots \ldots \ldots \ldots . . .40$

2.1.10.2. Procedimiento para la detección de operaciones inusuales (Seguimiento $\quad$......... 40 transaccional)

2.1.11. $\quad$ Determinación y reporte de operaciones sospechosas $\quad$................... 41

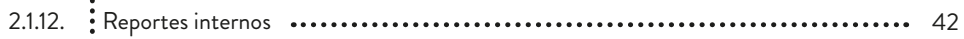

2.1.12.1. Reporte interno de transacciones sospechosas o inusuales $\quad$................ 42

2.1.12.2. Reporte interno de la etapa de monitoreo $\quad$.............................. 43

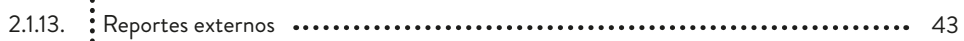

2.1.13.1. Reporte de operaciones sospechosas (ROS) $\quad \ldots \ldots \ldots \ldots \ldots \ldots \ldots \ldots \ldots \ldots \ldots \ldots . \ldots \ldots$

2.1.13.2. Reporte de transacciones en efectivo $\quad \ldots \ldots \ldots \ldots \ldots \ldots \ldots \ldots \ldots \ldots \ldots \ldots \ldots \ldots \ldots . \ldots \ldots$

2.1.13.3. Reporte de clientes exonerados $\quad$..................................... 44

2.1.13.4. Reporte sobre operaciones de transferencia, remesa, compra y venta de divisas $\ldots . . .45$

2.1.13.5. Reporte sobre productos ofrecidos por las empresas vigiladas $\quad \ldots \ldots \ldots \ldots \ldots \ldots \ldots . . .45$

2.1.13.6. Reporte de información sobre campañas y partidos políticos $\quad$................ 45

UNIDAD III POLÍTICAS SARLAFT Pág.

3.1. $\quad$ : Políticas generales $\quad$ (................................................. 46

3.2. Políticas para cada uno de los elementos del SARLAFT $\quad \ldots \ldots \ldots \ldots \ldots \ldots \ldots \ldots \ldots \ldots$

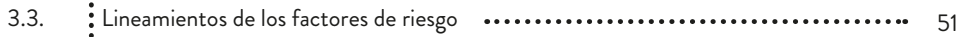

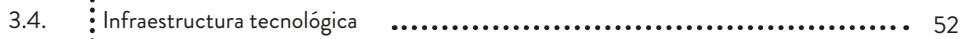

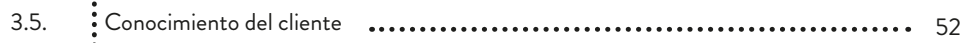

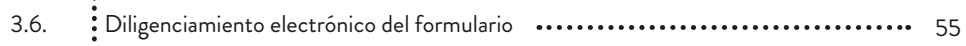

3.7. $\quad \vdots$ Vinculación de personas autorizadas para impartir órdenes y firmar a nombre de $\quad \ldots . . .55$

3.8. $\quad$ Requisitos en materia de documentación anexa al proceso de vinculación. ........... 55

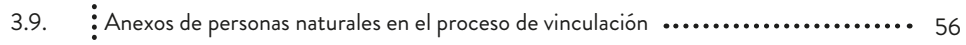

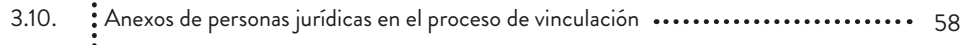

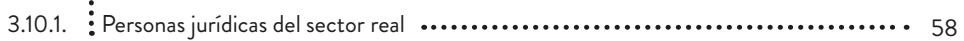




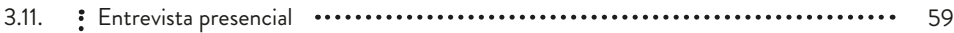

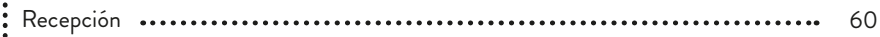

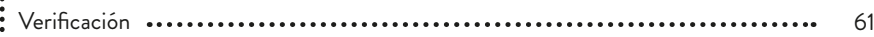

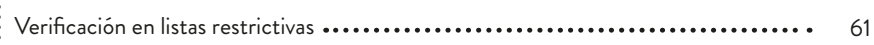

3.16. Recomendación de actualización de la información............................ 62

3.17. Clientes que pueden exponer un mayor grado de riesgo de LA/FT............... 62

3.18. Vinculación de clientes catalogados como personas públicamente expuestos ....... 63

3.19. Seguimiento de los clientes catalogados como personas públicamente expuestas .... 63

3.20. Cumplimiento de las recomendaciones del GAFISUP (Grupo de acción financiera .. 64 de América Latina)

3.21. Atención de requerimientos de autoridades competentes $\ldots \ldots \ldots \ldots \ldots \ldots \ldots \ldots \ldots$

3.22. $\quad$ Lineamientos para la prevención y resolución de conflictos de interés $\quad \ldots \ldots \ldots \ldots .65$

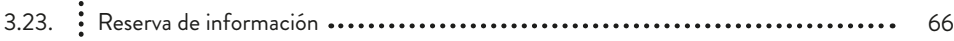

3.24. $\quad$ Empleados autorizados para consultar los documentos relativos a las etapas y ....... 67

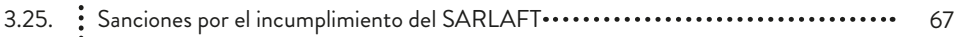

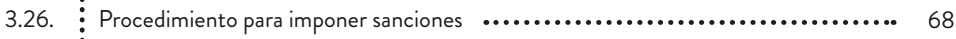

3.27. Recomendaciones de seguimiento a operaciones realizadas por usuarios $\ldots . . . . . . .68$

3.28. Controles sugeridos para el pago de giros o transferencias en establecimiento de $\quad$.... 69

\section{UNIDAD IV ESTRUCTURA ORGANIZACIONAL EN MATERIA DEL SARLAFT Pág.}

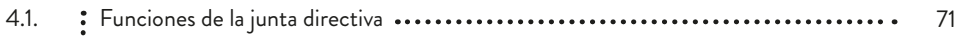

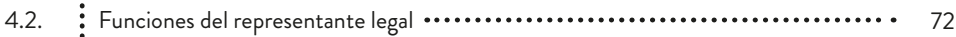

4.3. Requisitos y funciones del oficial de cumplimiento $\quad \ldots \ldots \ldots \ldots \ldots \ldots \ldots \ldots \ldots \ldots \ldots$

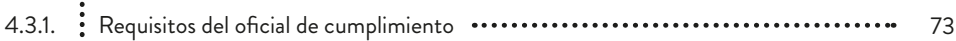

4.3.2. Funciones del oficial de cumplimiento $\ldots \ldots \ldots \ldots \ldots \ldots \ldots \ldots \ldots \ldots \ldots \ldots \ldots \ldots \ldots . \ldots \ldots$

4.3.3. Obligaciones de los empleados y prestadores de servicios en materia de SARLAFT .. 75

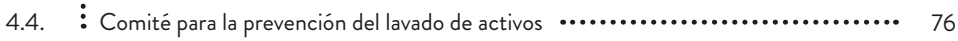




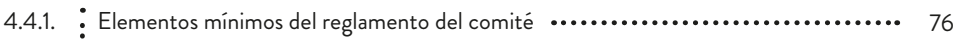

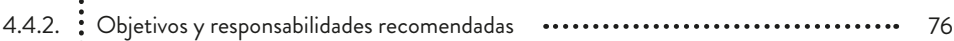

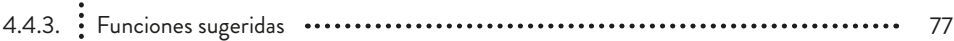

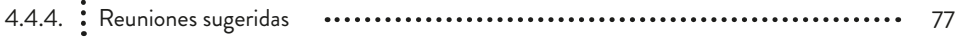

\section{UNIDAD V ÓRGANOS DE CONTROL Pág.}

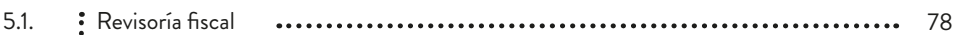

5.2. $\quad$ Auditoría interna o quien ejecute funciones similares o haga sus veces $\ldots . \ldots \ldots \ldots .78$

UNIDAD VI CONCLUSIONES Pág.

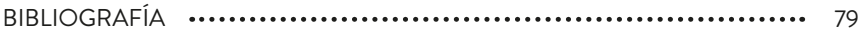

TABLA DE FIGURAS Pág.

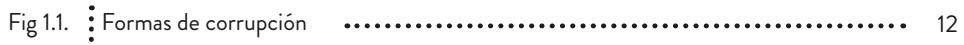

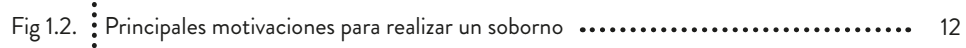

Fig 1.3. : Elementos negativos que fomentan el subdesarrollo económico y promueven la ...... 13 delincuencia en las instituciones

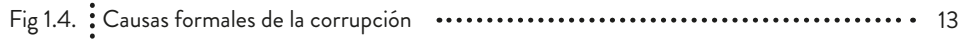

Fig 1.5. Causas materiales de la corrupción $\quad$.................................... 14

Fig 1.6. $\vdots$ Estructura penal del LA/FT $\quad$....................................... 15

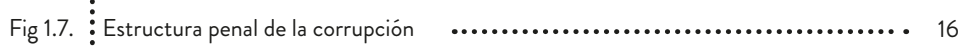

Fig 1.8. Línea del tiempo del marco normativo anticorrupción en Colombia $\quad$............. 16

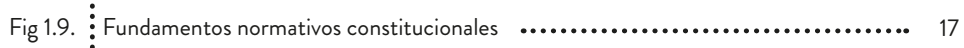

Fig 1.10. Ley 190 de $1995 \quad$....................................................... 17

Fig 1.11. Convención Interamericana contra la corrupción $1996 \quad$......................... 18

Fig 1.12. Convención de las Naciones Unidas contra la corrupción 2003 ............... 18

Fig 1.13. Estatuto anticorrupción Colombia $2011 \quad$..................................... 19

Fig 2.1. : Etapas del SARLAFT $\quad$.

Fig 2.2. Características para realizar análisis de actividades de clientes $\ldots \ldots \ldots \ldots \ldots \ldots \ldots \ldots .22$

Fig 2.3. Metodología KDD $\quad$............................................ 23






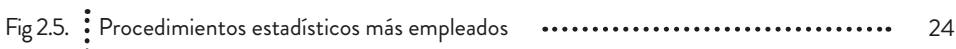

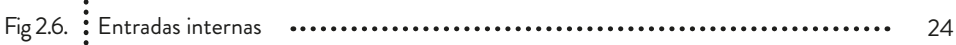

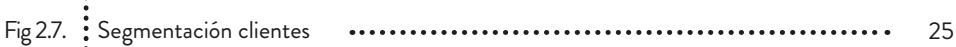

Fig 2.8. $\begin{aligned} & \text { Proporción de encuestados que perciben ciertas actividades como vulnerables } \\ & \cdots\end{aligned}$ 2017- 2018

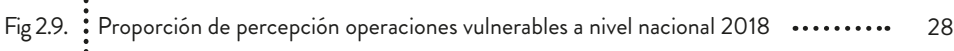

Fig 2.10. Proporción de percepción de servicios vulnerables a nivel nacional $2018 \quad \ldots \ldots \ldots \ldots 29$

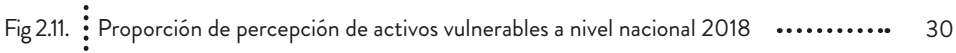

Fig 2.12. Proporción de percepción de cumplimiento e implementación de mecanismos de $\ldots \quad$.. 31 prevención y detección de LA/FT 2017 - 2018


terrorismo

Fig 2.14. Procedimientos para definir las medidas de control $\quad$.......................... 34

Fig 2.15. Monitoreo del riesgo de lavado de activos y financiación del terrorismo..........$\quad 35$

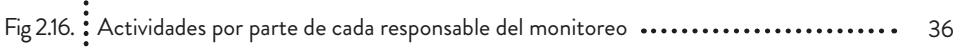

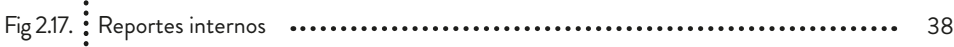

Fig $2.18 \vdots$ Variables para la segmentación $\quad$.......................................... 39

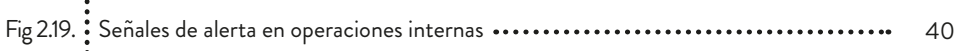

Fig 2.20 Otros tipos de alerta $\quad$................................................. 41

Fig 2.21. Contenido de reporte de operación sospechosa (ROS) $\quad$..................... 43

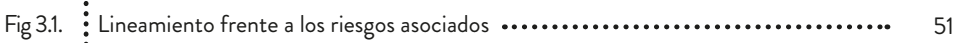

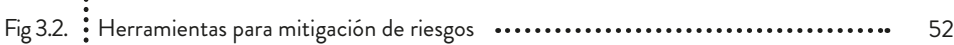

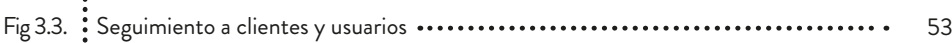

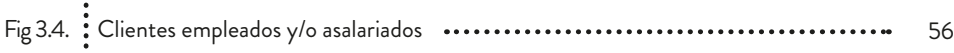

Fig 3.5.

Fig 3.6. Menores de edad o personas que dependen económicamente de un tercero $\ldots . . . . . \quad 57$

Fig 3.7. $\vdots$ Independientes $\quad$................................................... 57

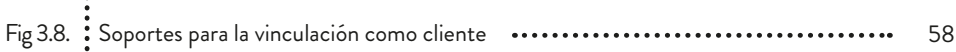

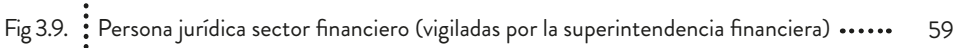

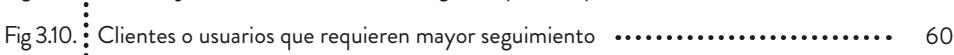

Fig 3.11. Contenido básico del formulario de visita a domicilio del cliente $\ldots . . . . . . . . . . . .60$ 


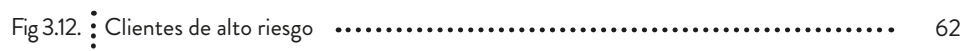

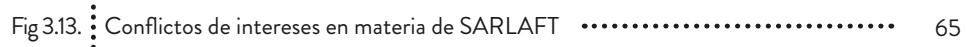

Fig 3.14.

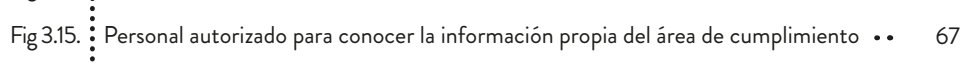

Fig 3.16. $\vdots$ Elementos mínimos del reglamento del comité $\quad$............................. 76

TABLA DE CUADROS Pág.

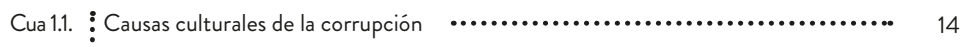

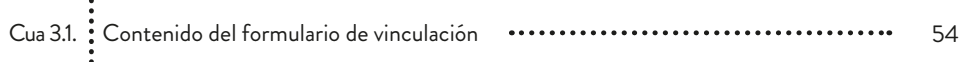



